Int. J. Electrochem. Sci., 13 (2018) 10894 - 10903

\title{
Development of a New Potentiometric Sensor based on home made Iodide ISE Enriched with ZnO Nanoparticles and its Application for Determination of Penicillamine
}

\author{
Ante Prkićl, ${ }^{1,}$, Tina Vukušić ${ }^{2}$, Josipa Giljanović ${ }^{1}$, Vesna Sokol ${ }^{3}$, Perica Bošković ${ }^{4}$, \\ Magdy Lučić Lavčević ${ }^{5}$, Ivana Mitar ${ }^{4}$, Miće Jakić ${ }^{6}$ \\ ${ }^{1}$ Department of Analytical Chemistry, Faculty of Chemistry and Technology, University of Split, \\ Ruđera Boškovića 35, 21000 Split, Croatia. \\ ${ }^{2}$ Faculty of Chemistry and Technology, University of Split, Ruđera Boškovića 35, 21000 Split, \\ Croatia. \\ ${ }^{3}$ Department of Physical Chemistry, Faculty of Chemistry and Technology, University of Split, \\ Ruđera Boškovića 35, 21000 Split, Croatia. \\ ${ }^{4}$ Department for Chemistry, Faculty of Science, University of Split, Ruđera Boškovića 33, 21000 \\ Split, Croatia. \\ ${ }^{5}$ Department of Physics, Faculty of Chemistry and Technology, University of Split, Ruđera Boškovića \\ 35, 21000 Split, Croatia. \\ ${ }^{6}$ Department of Organic Technology, Faculty of Chemistry and Technology, University of Split, \\ Ruđera Boškovića 35, 21000 Split, Croatia. \\ *E-mail: prkic@ktf-split.hr
}

doi: $10.20964 / 2018.11 .92$

Received: 3 July 2018 / Accepted: 31 August 2018 / Published: 1 October 2018

This paper describes the preparation and application of "home-made" iodide ISE enriched with $\mathrm{ZnO}$ nanoparticles. Iodide ISE membrane was made of $\mathrm{AgI}: \mathrm{Ag}_{2} \mathrm{~S}: \mathrm{PTFE}=1: 1: 2$ and enriched with $\mathrm{ZnO}$ nanoparticles in ratio 1-5.0 wt.\%. Prepared membranes were used for penicillamine (Pen) determination in acetic buffer $(\mathrm{pH}=4-4.75)$ and in perchloric acid $(\mathrm{pH}=1-2)$. Due to the low price method, simplicity and relative speed, a possibility for Pen determination was found. Pen determination showed better results in an acetic buffer, $\mathrm{pH}=4$ than in perchloric acid. All experiments for Pen measurements were done without pretreatment of pharmaceuticals. The determination is based on the reaction between Pen with both $\mathrm{Ag}^{+}$and $\mathrm{Zn}^{2+}$ from the electrode membrane. A newly described method has linear response range for Pen $2.45 \times 10^{-6}-1 \times 10^{-2} \mathrm{~mol} \mathrm{~L}^{-1}$ and a detection limit of $2.24 \times 10^{-6}$ $\mathrm{mol} \mathrm{L}{ }^{-1}$. The found concentrations of Pen are in a very good agreement with the declared ones with the standard deviation of $2.70 \%$.

Keywords: penicillamine, potentiometric, determination, "home-made" ion-selective electrode, nanoparticles 


\section{FULL TEXT}

(C) 2018 The Authors. Published by ESG (www.electrochemsci.org). This article is an open access article distributed under the terms and conditions of the Creative Commons Attribution license (http://creativecommons.org/licenses/by/4.0/). 